This item was submitted to Loughborough's Research Repository by the author.

Items in Figshare are protected by copyright, with all rights reserved, unless otherwise indicated.

\title{
Measuring muscle size and symmetry in healthy adult males using a time- efficient analysis of magnetic resonance images
}

PLEASE CITE THE PUBLISHED VERSION

https://doi.org/10.1088/1361-6579/ab2323

\section{PUBLISHER}

(C) Institute of Physics and Engineering in Medicine. Published by IOP Publishing

\section{VERSION}

AM (Accepted Manuscript)

\section{PUBLISHER STATEMENT}

This is the Accepted Manuscript version of an article accepted for publication in Physiological Measurement. IOP Publishing Ltd is not responsible for any errors or omissions in this version of the manuscript or any version derived from it. The Version of Record is available online at https://doi.org/10.1088/1361-6579/ab2323.

\section{LICENCE}

CC BY-NC-ND 4.0

\section{REPOSITORY RECORD}

Rothwell, Daniel, David Williams, and Laura-Anne Furlong. 2019. "Measuring Muscle Size and Symmetry in Healthy Adult Males Using a Time-efficient Analysis of Magnetic Resonance Images". figshare. https://hdl.handle.net/2134/37841. 
Authors:

Daniel T Rothwell ${ }^{1}$, David J Williams ${ }^{2}$, Laura-Anne M Furlong ${ }^{1,3 *}$

Title:

Measuring muscle size and symmetry in healthy adult males using a time-efficient analysis of Magnetic Resonance images

Affiliations: $\quad{ }^{1}$ School of Sport, Exercise and Health Sciences, Loughborough University, Loughborough, Leicestershire, United Kingdom.

${ }^{2}$ Wolfson School of Mechanical, Electrical and Manufacturing Engineering, Loughborough University, Loughborough, Leicestershire, United Kingdom.

${ }^{3}$ National Centre for Sport and Exercise Medicine - East Midlands, Loughborough, Leicestershire, United Kingdom.

*Correspondence: Laura-Anne M. Furlong, BSc., PhD

School of Sport, Exercise and Health Sciences

Loughborough University

Epinal Way

Loughborough

Leicestershire

LE11 3TU

United Kingdom

E: L.A.M.Furlong@1boro.ac.uk

P: +44 (0) 1509223059

Author ORCID: $\quad$ Daniel T Rothwell: $\quad$ 0000-0002-5602-0217

David J Williams: 0000-0002-4943-8543

Laura-Anne M Furlong: 0000-0002-7303-4434 


\section{ABSTRACT}

Objective: Muscle volume (MV) analysis from magnetic resonance imaging (MRI) is time-intensive, and limited measurement reliability data are available. This study investigated a method to reduce lower limb MV analysis time demands, established reliability of these measurements, and applied the findings to quantify muscle size and symmetry in healthy adult males.

Approach: Bilateral MRI images were acquired from 15 healthy males (age: $26.5 \pm 4.6$ years, height: $1.81 \pm 0.09 \mathrm{~m}$, body mass: $80.4 \pm 12.4 \mathrm{~kg}$ ) for the entire lower limb. In two participants, the individual gluteals, quadriceps, hamstrings, and triceps surae were manually outlined every $5 \mathrm{~mm}$ and MV calculated using 5, 10, 15, 20, 25, and $30 \mathrm{~mm}$ distances between images to determine an appropriate distance for reducing analysis time. For all 15 participants, 35 muscles in each limb were manually outlined every 15 $\mathrm{mm}$ for use in MV calculations. Reliability of muscle cross-sectional area (CSA) measurements was determined within- and between-sessions and MV measurement reliability determined between-sessions. Between-limb symmetry was calculated using symmetry indices.

Main results: A $15 \mathrm{~mm}$ inter-slice distance was appropriate for measuring MV (mean difference compared to reference method: $0.7 \pm 0.7 \%$ ). Between-session measurement reliability was good for MV (Typical Error preferred kicking limb (TEP): 1.2\%, nonpreferred kicking limb (TENP): 0.8\%) and CSA (TEP: $3.4 \pm 2.9 \%$, $\mathrm{TE}_{\mathrm{NP}}: 3.2 \pm 1.9 \%$ ) although CSA typical error was larger with increased between-session time (TEP: 4.1 $\pm 3.1 \%$, TE $\mathrm{NP}: 4.7 \pm 4.0 \%$ ). Between-limb differences in MV were small (mean symmetry index: $0.4 \pm 4.1 \%$ ). Absolute differences in individual MV were larger (mean: $12.6 \pm 2.6 \%$ ), but representing muscles as functional anatomical groups showed smaller absolute between-limb differences (mean: $4.7 \pm 1.8 \%$ ).

Significance: MV analysis time demand can be reduced by increasing the distance between analysed MRI slices, although participant height, muscle length and shape require consideration. Minimal between-limb muscle size differences have been reported in adult males. 
KEYWORDS: lower limb, magnetic resonance imaging, measurement reliability, muscle volume, image processing, image analysis 


\section{INTRODUCTION}

Muscle size has the capacity to differentiate between healthy and clinical populations (Grimaldi et al. 2009), and between athletic cohorts (Bex et al. 2017). Loss of muscle with ageing is associated with reduced mobility and disease (Fuggle et al. 2017). The ability to reliably quantify muscle size, and monitor changes over time and between individuals, has a valuable application for clinicians and researchers in prescribing and evaluating patient-specific injury prevention, rehabilitation, and training programmes. Baseline measurements of typical muscle size and between-limb differences can inform musculoskeletal models and engineering design in support of these applications. Previous work focused on the entire lower limb has established muscle size unilaterally in healthy males and females (Handsfield et al. 2014), and bilaterally in collegiate sprinters (Handsfield et al. 2017) and athletes from sports with a limb preference (Tate et al. 2006). However, how muscle size and symmetry varies throughout the lower limb is currently unknown in the general, recreationally active population, limiting its broader potential application.

Muscle volume (MV) is commonly used to represent muscle size as it remains reasonably constant in the absence of training induced hypertrophy, disease related atrophy, or injury. It is typically calculated by manually outlining muscle crosssectional areas (CSA) on axial images obtained using Magnetic Resonance Imaging (MRI; Bex et al. 2017, Grimaldi et al. 2009, Handsfield et al. 2014, 2017, Nordez et al. 2009, Tate et al. 2006). The reliability of this approach has been established in the quadriceps, hamstrings, gracilis, sartorius, and triceps surae in athletes from sports with a leg preference (Tate et al. 2006), and throughout the lower limb in typically developed children and children with cerebral palsy (Handsfield et al., 2016). However, there are limited data available throughout the lower limb in adult populations, attributable in part to the intensive time demand of completing this type of analysis. Typical analysis time has been reported as 25 hours per limb when analysing $5 \mathrm{~mm}$ thick slices with 0 $\mathrm{mm}$ inter-slice spacing (Handsfield et al. 2014). Reducing the required analysis time could increase the volume of MRI-derived research outputs in this area and develop existing knowledge of the musculoskeletal system. Increasing the inter-slice distance used for analysis (i.e. rather than analysing all slices of MRI data acquired, images are analysed with 10/15/20 mm gaps between them) could potentially assist in this regard. 
In the quadriceps, an inter-slice distance of $15 \mathrm{~mm}$ has been used pragmatically in recent research (Balshaw et al., 2017) and an inter-slice distance of 30 to $36 \mathrm{~mm}$ has previously been considered appropriate (Nordez et al. 2009), whereas an inter-slice distance of $6 \mathrm{~mm}$ has been used for quantifying gluteal MV (Grimaldi et al., 2009). To date, the percentage difference observed with increasing inter-slice distance has not been reported for all lower limb muscles of interest (such as the hip extensors, knee flexors and extensors, and ankle plantarflexors), and so evidence-based selection of inter-slice distance has not been possible.

Muscle size is a key physiological variable used in the design and monitoring of injury prevention, training, and rehabilitation programmes, and for informing musculoskeletal models and design of assistive devices. Large bilateral data sets are time-intensive to obtain, and establishing an accurate, reliable method of reducing time analysis demands would be beneficial for both researchers and practitioners using this type of data. The aim of this study was to investigate the application of MRI for the measurement of bilateral lower limb muscle size and symmetry in a group of healthy adult males. Specific objectives were to explore the differences observed in MV measurements when increasing inter-slice distance to reduce the analysis time, establish the measurement reliability of manual MRI image analysis, and use these findings to quantify typical muscle size and muscle size symmetry in healthy adult males.

\section{METHODS}

\section{Participants}

Following University ethical approval, 15 healthy Caucasian males gave written informed consent to participate (age: $26.5 \pm 4$.6 years, height: $1.81 \pm 0.09 \mathrm{~m}$, body mass: $80.4 \pm 12.4 \mathrm{~kg}$ ). All participants performed at least 30 minutes of physical activity three times per week, had no history of lower limb surgery, and were free from injury for six months prior to testing. Participants regularly involved in sports with a limb preference (e.g. field hockey, soccer) were excluded from data collection to prevent potential bias of results. Limb preference (preferred: P, non-preferred: NP) was defined as that used to kick a ball. Eleven participants preferred their right limb and four their left. 


\section{MRI data acquisition}

Bilateral axial spin-echo T1-weighted MRI images were acquired from the iliac crest to the base of the distal calcaneus using 3-Tesla MRI (Discovery MR750w, GE Healthcare, Buckinghamshire, UK). Images were acquired in three to four scanning blocks of 58 to 100 slices depending on participant height. Block overlap was identified using fish oil capsule references which were visible on the images used in the analysis. Field of view $(512 \mathrm{~mm} \times 512 \mathrm{~mm})$, axial in-plane resolution $(0.9 \mathrm{~mm} \times 0.9 \mathrm{~mm})$, flip angle $\left(111^{\circ}\right)$, slice thickness $(5 \mathrm{~mm})$ and inter-slice spacing $(0 \mathrm{~mm})$ remained constant across participants, and echo time $(\mathrm{TE}=7.2-8 \mathrm{~ms})$ and repetition time $(\mathrm{TR}=506-$ $850 \mathrm{~ms}$ ) varied. Participants were instructed to minimise movement as much as possible while images were obtained to reduce potential artefact. Total scan time was approximately 20 minutes per participant.

\section{Data processing - inter-slice distance analysis}

MRI images were analysed in OsiriX Lite (v.8.0.1, Pixmeo, Geneva, Switzerland) open source software by one investigator. Brightness and zoom tools were used to improve tissue contrast and enable muscle boundaries to be better identified, with reference to an online MRI resource (Bennett 2016). For the inter-slice distance analysis, the gluteals (gluteus maximus, medius, minimus), quadriceps (rectus femoris, vastus intermedialis, lateralis, medialis), hamstrings (semitendinosus, semimembranosus, biceps femoris long and short head) and triceps surae (medial and lateral gastrocnemius, soleus) of three participants, representative of small (height: $1.73 \mathrm{~m}$ ), medium (height: $1.82 \mathrm{~m}$ ), and large (height: $1.90 \mathrm{~m}$ ) heights in the current sample, were manually outlined in both limbs on every slice, providing a digital representation of CSA and Cartesian coordinates of the boundary points. These coordinates were used to reconstruct the three-dimensional shape of each muscle using Delaunay triangulation in OsiriX Lite, providing a reference measurement of MV. Delaunay triangulation represented the three-dimensional muscle shape with an average of 951 points for the smallest muscle, gluteus minimus (i.e. 4.7 points per $\mathrm{cm}^{2}$ on each axial image), and 4767 points for the larger vastus lateralis (i.e. 3.4 points per $\mathrm{cm}^{2}$ on each axial image). Inter-slice distances of 5, 10, 15, 20, 25, and $30 \mathrm{~mm}$ were evaluated against this by 
expressing the absolute difference between the calculated and the reference MV as a percentage of the reference MV. MV was calculated in centimetres cubed $\left(\mathrm{cm}^{3}\right)$ using:

$$
\sum_{i=1 \ldots n-1}^{n}\left(\frac{C S A_{i}+C S A_{i+1}}{2}\right) \times h
$$

Where $C S A_{i}$ is CSA at slice $i, C S A_{i+1}$ is CSA at slice $i+1, h=$ distance between slices, $n=$ total number of slices in the muscle. MV was normalised to the product of body mass and height $\left(\mathrm{cm}^{3} / \mathrm{kg} \cdot \mathrm{m}\right)$ to account for differences in body size (Handsfield et al. 2014). The MV calculation presented above was compared to the use of a cubic spline interpolation to check using a subgroup of the sample $(n=2)$ differences in estimated muscle volume by method. After spline fitting, intermediate CSA at $1 \mathrm{~mm}$ intervals were then summed to calculate MV. Small and acceptable differences were found between the two methods, ranging from $0.1 \pm 0.0 \%$ for biceps femoris long head to 3.2 $\pm 3.4 \%$ for gluteus medius. The equation shown above was hence used to calculate all MV.

\section{Measurement reliability}

The reliability of segmenting muscle CSA was established within- and betweensessions. Due to the time demand of the analysis (approximately 24 hours per participant), MV reliability was determined between-sessions in two participants (height: $1.93 \mathrm{~m}$ and $1.75 \mathrm{~m}$ ) by re-conducting the analysis after a minimum of sevendays, with all muscles analysed in the same order. Cross-sectional area reliability was determined within- and between-sessions for all 15 participants, bilaterally at four sites; $25 \%$ and $75 \%$ of thigh (greater trochanter to lateral femoral epicondyle) and shank length (medial knee joint line to medial malleolus), to maximise the number of muscles available that were not at their origin or insertion. The image number was recorded in the first session and re-analysed in all subsequent sessions to ensure the analysis evaluated the reliability of measuring CSA without being limited by potential error in identifying the measurement site. Cross-sectional areas were included in reliability analyses when larger than $1.0 \mathrm{~cm}^{2}$ in at least eight participants to remove outliers and reduce the possibility of bias. Cross-sectional areas were deleted and repeated 
immediately for within-session reliability evaluations and after 12 hours and six months for between-session reliability evaluations.

\section{Establishing normative muscle size and symmetry}

The inter-slice distance analysis identified $15 \mathrm{~mm}$ as an appropriate inter-slice distance and CSA of 35 muscles in each limb were outlined at this interval, from origin to insertion, starting from the iliac crest and moving distally, in all 15 participants. Where the final inter-slice distance was less than $15 \mathrm{~mm}$, the value of $h$ in the MV calculation was adjusted accordingly. Due to their short length (mean length P: $4.3 \pm 2.7 \mathrm{~cm}$, NP: $4.2 \pm 2.7 \mathrm{~cm}$ ) the small hip external rotators (piriformis, gemellus superior and inferior, obturator internus and externus, quadratus femoris) were analysed on every slice. For all muscles, the analysis was completed on the same day it was started. Calculated CSA were visually reviewed for accuracy by the same investigator prior to MV calculation.

Individual MV were summed to calculate functional anatomical group volumes (i.e. muscles performing the same agonistic actions, such as hip flexors and extensors, as defined by Floyd, 2007). This was preferred to using group CSA as preliminary analysis in one participant identified systematic overestimation of MV throughout the lower limb using this approach, due to the inclusion of inter-muscular adipose tissue (mean difference versus summed individual volumes P: $8.3 \pm 3.6 \%$, NP: $10.9 \pm 3.5 \%$ ).

Symmetry index (SI; Karamanidis et al. 2003) was used to quantify the magnitude and direction of between-limb differences in individual and group MV as a percentage of the mean value of both limbs:

$$
S I=\frac{\left(M V_{P}-M V_{N P}\right)}{\frac{1}{2}\left(M V_{P}+M V_{N P}\right)} \times 100 \%
$$

Where $M V_{P}$ is preferred limb muscle volume and $M V_{N P}$ is non-preferred limb muscle volume. The absolute value of the SI (absolute symmetry index, ASI) was also calculated to account for positive and negative SI cancelling each other out in group means. 
To quantify the size of each participant's individual MV relative to the study population, individual MV were normalised to the group mean and standard deviation:

$$
\text { z-score }_{M V_{i}}\left(P^{j}\right)=\frac{\left(P^{j}{ }_{M V_{i}}-\mu_{M V_{i}}\right)}{\sigma_{M V_{i}}}
$$

Where z-score is relative MV, $M V_{i}$ is volume of muscle $i, P^{j}$ is participant $j$, and $\mu_{M V i}$ and $\sigma_{M V i}$ are the group mean and standard deviation, respectively, for $M V_{i}$. Positive and negative z-scores were used to indicate when $M V_{i}$ was larger or smaller than the group mean, respectively.

\section{Statistical analysis}

All computational analyses were completed in Microsoft Excel 2010 (Microsoft Inc., Redmond, WA., USA). All statistical analyses were conducted in IBM SPSS Statistics 23 (IBM Corporation, Armonk, NY, USA). The alpha level for statistical significance was set to $\mathrm{p} \leq 0.05$.

MV and CSA measurement reliability was evaluated using percentage differences:

$$
\frac{\mid \text { sample } 1-\text { sample } 2 \mid}{\text { sample } 1} \times 100 \%
$$

Typical Error (TE) was calculated as:

$$
T E=\frac{S D_{\text {differences }}}{\sqrt{2}}
$$

Where $S D_{\text {differences }}$ is the standard deviation of the differences between samples. Twoway mixed measures single intra-class correlation coefficients (ICC) with absolute agreement were used to evaluate within- and between-session measurement reliability. Relative agreement within- and between-sessions was evaluated using Pearson's 
correlation coefficient. The practical significance of the magnitude of differences in CSA within- and between-sessions was evaluated using Cohen (1988) $d_{z}$ effect sizes:

$$
d_{z}=\frac{m_{z}}{\sigma_{z}}
$$

Where $m_{z}$ is the absolute difference between sample means and $\sigma_{z}$ is the pooled standard deviation of both samples. Effect sizes were considered practically significant when larger than 0.6 (i.e. moderate according to Hopkins et al. 2009).

The statistical and practical significance of between-limb differences in individual and group MV were evaluated using paired samples t-tests and effect sizes, respectively.

\section{RESULTS}

\section{Inter-slice distance}

For all muscles the smallest mean absolute percentage difference in MV compared to the reference method was found with an inter-slice distance of $5 \mathrm{~mm}$ (mean difference: $0.3 \pm 0.5 \%$ ). This percentage difference and variability of the differences across muscles increased with increasing inter-slice distances (mean absolute percentage difference for all muscles at $10 \mathrm{~mm}: 0.6 \pm 0.6 \%, 15 \mathrm{~mm}: 0.7 \pm 0.7 \%, 20 \mathrm{~mm}: 0.9 \pm 1.0 \%, 25 \mathrm{~mm}: 1.2$ $\pm 1.5 \%, 30 \mathrm{~mm}: 1.3 \pm 1.5 \%$ ) and values for individual muscles are shown in Table 1 . The considerable time-saving and low absolute percentage differences found across muscles with an inter-slice distance of $15 \mathrm{~mm}$, together with its applicability for use in shorter muscles, supported its appropriateness for application throughout the lower limb, excluding the small hip external rotators which were analysed on every slice due to their short length. 


\section{Table 1}

Mean \pm standard deviation (range) of absolute percentage differences (\%) in muscle volume for 5, 10, 15, 20, 25, and $30 \mathrm{~mm}$ inter-slice distances compared to the reference method (Delaunay triangulation) in three participants with heights of $1.73 \mathrm{~m}, 1.82 \mathrm{~m}$, and $1.90 \mathrm{~m}$.

\begin{tabular}{|c|c|c|c|c|c|c|c|}
\hline $\begin{array}{l}\text { Muscle } \\
\text { group }\end{array}$ & Muscle & $5 \mathrm{~mm}(\%)$ & 10 mm (\%) & $15 \mathrm{~mm}(\%)$ & $20 \mathrm{~mm}(\%)$ & $25 \mathrm{~mm}(\%)$ & $30 \mathrm{~mm}(\%)$ \\
\hline \multicolumn{2}{|c|}{$\begin{array}{l}\text { Approximate analysis time for both legs } \\
\text { of one participant (hours) }\end{array}$} & 24.0 & 12.0 & 8.0 & 6.0 & 4.8 & 4.0 \\
\hline \multirow{4}{*}{ Gluteals } & Gluteus maximus & $0.0 \pm 0.0(0.0$ to 0.1$)$ & $0.1 \pm 0.1(0.0$ to 0.3$)$ & $0.4 \pm 0.3(0.2$ to 1.0$)$ & $0.5 \pm 0.3(0.1$ to 0.9$)$ & $0.7 \pm 0.5(0.1$ to 1.6$)$ & $0.9 \pm 0.5(0.1$ to 1.3$)$ \\
\hline & Gluteus medius & $0.0 \pm 0.0(0.0$ to 0.0$)$ & $0.7 \pm 0.7(0.0$ to 1.8$)$ & $0.8 \pm 0.5(0.2$ to 1.6$)$ & $1.6 \pm 1.4(0.2$ to 4.3$)$ & $2.5 \pm 2.7(0.1$ to 7.7$)$ & $2.3 \pm 2.0(0.7$ to 6.3$)$ \\
\hline & Gluteus minimus & $0.0 \pm 0.0(0.0$ to 0.0$)$ & $1.5 \pm 0.6(0.8$ to 2.3$)$ & $2.2 \pm 1.0(0.8$ to 3.4$)$ & $1.7 \pm 0.7(0.4$ to 2.4$)$ & $3.4 \pm 3.0(0.5$ to 8.0$)$ & $4.4 \pm 2.9(0.1$ to 8.7$)$ \\
\hline & Mean & $0.0 \pm 0.0(0.0$ to 0.1$)$ & $0.8 \pm 0.7(0.0$ to 2.3$)$ & $1.1 \pm 0.9(0.2$ to 3.4$)$ & $1.3 \pm 0.6(0.1$ to 4.3$)$ & $2.2 \pm 1.3(0.1$ to 8.0$)$ & $2.5 \pm 1.7(0.1$ to 8.7$)$ \\
\hline \multirow{5}{*}{ Quadriceps } & Vastus medialis & $0.5 \pm 0.6(0.1$ to 1.7$)$ & $0.6 \pm 0.5(0.1$ to 1.5$)$ & $0.4 \pm 0.3(0.0$ to 0.8$)$ & $1.0 \pm 0.4(0.5$ to 1.5$)$ & $0.9 \pm 0.3(0.6$ to 1.4$)$ & $0.7 \pm 0.5(0.1$ to 1.7$)$ \\
\hline & Vastus lateralis & $0.4 \pm 0.6(0.0$ to 1.6$)$ & $0.5 \pm 0.7(0.0$ to 1.9$)$ & $0.7 \pm 0.6(0.1$ to 1.7$)$ & $0.8 \pm 0.8(0.0$ to 2.1$)$ & $0.6 \pm 0.4(0.2$ to 1.2$)$ & $1.1 \pm 0.6(0.1$ to 1.7$)$ \\
\hline & Vastus intermedialis & $0.3 \pm 0.6(0.0$ to 1.6$)$ & $0.5 \pm 0.7(0.0$ to 1.9$)$ & $0.7 \pm 0.7(0.1$ to 2.0$)$ & $0.8 \pm 0.9(0.0$ to 2.4$)$ & $0.7 \pm 0.5(0.1$ to 1.4$)$ & $0.6 \pm 0.6(0.1$ to 1.5$)$ \\
\hline & Rectus femoris & $0.1 \pm 0.2(0.0$ to 0.6$)$ & $0.4 \pm 0.3(0.1$ to 0.9$)$ & $0.3 \pm 0.3(0.0$ to 0.9$)$ & $0.2 \pm 0.1(0.0$ to 0.3$)$ & $0.6 \pm 0.3(0.2$ to 1.1$)$ & $0.7 \pm 0.7$ ( 0.0 to 1.8$)$ \\
\hline & Mean & $0.3 \pm 0.2(0.0$ to 1.7$)$ & $0.5 \pm 0.1(0.0$ to 1.9$)$ & $0.5 \pm 0.2(0.0$ to 2.0$)$ & $0.7 \pm 0.4(0.0$ to 2.4$)$ & $0.7 \pm 0.1(0.1$ to 1.4$)$ & $0.8 \pm 0.2(0.0$ to 1.8$)$ \\
\hline \multirow{5}{*}{ Hamstrings } & Semitendinosus & $0.3 \pm 0.7$ (0.0 to 1.7$)$ & $0.5 \pm 0.5(0.2$ to 1.5$)$ & $0.4 \pm 0.5(0.1$ to 1.3$)$ & $0.7 \pm 0.7(0.1$ to 2.0$)$ & $0.5 \pm 0.6(0.0$ to 1.7$)$ & $0.8 \pm 0.4(0.2$ to 1.5$)$ \\
\hline & Long head biceps femoris & $0.6 \pm 0.9(0.0$ to 2.4$)$ & $0.7 \pm 0.8(0.1$ to 2.3$)$ & $0.7 \pm 0.8(0.1$ to 2.3$)$ & $0.7 \pm 0.9(0.2$ to 2.5$)$ & $0.9 \pm 0.8(0.1$ to 2.2$)$ & $0.9 \pm 1.1(0.1$ to 2.8$)$ \\
\hline & Short head biceps femoris & $0.8 \pm 0.7(0.0$ to 1.9$)$ & $0.9 \pm 0.5(0.2$ to 1.4$)$ & $0.8 \pm 1.0(0.0$ to 2.5$)$ & $1.2 \pm 1.1(0.0$ to 3.0$)$ & $0.8 \pm 0.5(0.1$ to 1.4$)$ & $1.4 \pm 0.8(0.4$ to 2.7$)$ \\
\hline & Semimembranosus & $0.4 \pm 0.4(0.0$ to 1.1$)$ & $0.4 \pm 0.4(0.0$ to 1.0$)$ & $0.5 \pm 0.5(0.0$ to 1.4$)$ & $0.6 \pm 0.7(0.1$ to 1.8$)$ & $0.9 \pm 1.0(0.1$ to 2.7$)$ & $0.7 \pm 0.6(0.3$ to 2.0$)$ \\
\hline & Mean & $0.5 \pm 0.2(0.0$ to 2.4$)$ & $0.6 \pm 0.2(0.0$ to 2.3$)$ & $0.6 \pm 0.2(0.0$ to 2.5$)$ & $0.8 \pm 0.3(0.0$ to 3.0$)$ & $0.8 \pm 0.2(0.0$ to 2.7$)$ & $0.9 \pm 0.3(0.1$ to 2.8$)$ \\
\hline \multirow{3}{*}{ Triceps surae } & Medial gastrocnemius & $0.6 \pm 0.4(0.1$ to 1.2$)$ & $0.8 \pm 0.6(0.2$ to 1.6$)$ & $0.9 \pm 0.6(0.3$ to 1.7$)$ & $1.1 \pm 0.5(0.3$ to 1.6$)$ & $0.7 \pm 0.8(0.1$ to 2.2$)$ & $1.8 \pm 1.3(0.4$ to 3.7$)$ \\
\hline & Lateral gastrocnemius & $0.5 \pm 0.4(0.0$ to 1.1$)$ & $0.7 \pm 0.6(0.1$ to 1.5$)$ & $1.0 \pm 0.8(0.3$ to 2.1$)$ & $2.0 \pm 1.9(0.2$ to 4.9$)$ & $2.5 \pm 1.7(0.6$ to 4.6$)$ & $1.3 \pm 1.2(0.3$ to 3.5$)$ \\
\hline & Soleus & $0.2 \pm 0.1$ ( 0.0 to 0.4$)$ & $0.1 \pm 0.1(0.0$ to 0.3$)$ & $0.5 \pm 0.3(0.0$ to 0.8$)$ & $0.4 \pm 0.4(0.1$ to 1.1$)$ & $1.1 \pm 0.4(0.4$ to 1.5$)$ & $0.7 \pm 0.5(0.1$ to 1.4$)$ \\
\hline
\end{tabular}


Manual analysis of MRI images showed good intra-investigator reliability. Small percentage differences in CSA measurements were found within-sessions and betweensessions over 12 hours, although differences were larger over six months (Table 2) and in smaller muscles such as vastus medialis at $25 \%$ of thigh length in both limbs (mean difference P: $26.0 \pm 25.2 \%$, NP: $43.4 \pm 66.3 \%$ ). Individual muscle percentage differences are provided in Supplementary Material 1. ICC were high within-sessions (mean single measures ICC of all muscles P: $0.97 \pm 0.03$, NP: $0.97 \pm 0.03$ ), and betweensessions over 12-hours (mean single measures ICC of all muscles P: $0.96 \pm 0.03$, NP: $0.95 \pm 0.04$ ). Between-sessions over six months, ICC were high (mean single measures ICC of all muscles P: $0.92 \pm 0.09$, NP: $0.91 \pm 0.09$ ), with the exception of vastus medialis at $25 \%$ of thigh length in both limbs (ICC P: 0.63, NP: 0.62) and flexor digitorum longus of the preferred limb at $75 \%$ of shank length (ICC P: 0.65). Relative agreement between-sessions was strong across muscles over 12 hours and six months, with the lowest $r$ values being found for preferred limb extensor hallucis and digitorum longus at $25 \%$ of shank length $(r=0.90)$ and preferred limb tibialis posterior at $75 \%$ of shank length $(r=0.90)$. 
Table 2

Mean \pm standard deviation absolute percentage differences $(\%)$ in muscle cross sectional area within- and between-sessions. Individual muscle absolute percentage differences are presented in the supplementary information.

\begin{tabular}{|c|c|c|c|}
\hline Site & & Preferred limb $(\%)$ & Non-preferred limb (\%) \\
\hline \multirow{3}{*}{ Proximal thigh } & Within-session & $2.1 \pm 2.7$ & $1.3 \pm 2.0$ \\
\hline & Between-session (12 hours) & $1.9 \pm 1.7$ & $1.9 \pm 2.4$ \\
\hline & Between-session (6 months) & $7.7 \pm 6.7$ & $10.9 \pm 11.9$ \\
\hline \multirow{3}{*}{ Distal thigh } & Within-session & $2.4 \pm 3.0$ & $1.2 \pm 1.7$ \\
\hline & Between-session (12 hours) & $2.3 \pm 2.6$ & $1.4 \pm 2.2$ \\
\hline & Between-session (6 months) & $6.5 \pm 5.0$ & $6.2 \pm 4.8$ \\
\hline \multirow{3}{*}{ Proximal shank } & Within-session & $1.1 \pm 0.8$ & $1.0 \pm 0.9$ \\
\hline & Between-session (12 hours) & $1.3 \pm 0.8$ & $1.6 \pm 1.3$ \\
\hline & Between-session (6 months) & $5.8 \pm 2.5$ & $6.5 \pm 3.0$ \\
\hline \multirow{3}{*}{ Distal shank } & Within-session & $2.6 \pm 3.7$ & $2.2 \pm 2.3$ \\
\hline & Between-session (12 hours) & $3.8 \pm 3.6$ & $3.9 \pm 5.1$ \\
\hline & Between-session (6 months) & $9.0 \pm 4.5$ & $8.9 \pm 4.9$ \\
\hline
\end{tabular}


Compared to the first analysis session, minimal mean TE was found for CSA withinsessions (P: $0.2 \pm 0.1 \mathrm{~cm}^{2}, 3.1 \pm 2.7 \%$, NP: $0.2 \pm 0.1 \mathrm{~cm}^{2}, 2.8 \pm 2.8 \%$ ), and betweensessions over 12 hours (P: $0.3 \pm 0.2 \mathrm{~cm}^{2}, 3.4 \pm 2.9 \%$, NP: $0.2 \pm 0.2 \mathrm{~cm}^{2}, 3.2 \pm 1.9 \%$ ) and six months (P: $0.3 \pm 0.2 \mathrm{~cm}^{2}, 4.1 \pm 3.1 \%$, NP: $0.4 \pm 0.3 \mathrm{~cm}^{2}, 4.7 \pm 4.0 \%$ ). Higher TE was found within- and between-sessions for smaller CSA, such as flexor digitorum longus at $75 \%$ of shank length within-sessions (P: $0.2 \mathrm{~cm}^{2}, 13.9 \%, \mathrm{NP}: 0.2 \mathrm{~cm}^{2}, 13.8 \%$ ), and between-sessions over 12 hours (P: $0.3 \mathrm{~cm}^{2}, 14.2 \%$ ), and vastus medialis at $25 \%$ of thigh length between-sessions over six months (P: $0.9 \mathrm{~cm}^{2}, 14.8 \%, \mathrm{NP}: 1.2 \mathrm{~cm}^{2}$, 22.4\%). TE for individual muscles is provided in Supplementary Material 1.

Total MV showed good reliability in both limbs between-sessions. ICC were high (P: $>0.99$, NP: > 0.99), TE was low (P: $17.6 \mathrm{~cm}^{3}, 1.2 \%$, NP: $11.0 \mathrm{~cm}^{3}, 0.8 \%$ ) and relative agreement was good ( $\mathrm{r}$ P: > 0.99, NP: > 0.99). The mean absolute percentage difference in total MV between-sessions was high (mean absolute percentage difference P: 9.9 $\pm 12.4 \%$, NP: $10.7 \pm 13.3 \%$ ) due to large differences in smaller muscles, such as the small hip external rotators (mean absolute percentage difference P: $27.0 \pm 20.3 \%$, NP: $34.0 \pm 16.3 \%$ ), gluteus minimus (mean absolute percentage difference P: $10.3 \%$, NP: 9.8\%), adductor brevis (mean absolute percentage difference P: $21.0 \%$, NP: $11.3 \%$ ), popliteus (mean absolute percentage difference P: $14.3 \%$, NP: 10.6\%), flexor digitorum longus (mean absolute percentage difference P: 19.8\%, NP: 6.0\%), and flexor hallucis longus (mean absolute percentage difference P: 4.9\%, NP: 26.9\%, Table 3). Low between-session absolute percentage differences were found for functional anatomical groups (mean absolute percentage difference P: $2.2 \pm 1.9 \%$, NP: $2.9 \pm 4.2 \%$ ), with high ICC (P: > 0.99, NP: > 0.99), good relative agreement (r P: > 0.99, NP: > 0.99), and low TE (P: $17.6 \mathrm{~cm}^{3}, 1.2 \%$, NP: $11.0 \mathrm{~cm}^{3}, 0.8 \%$ ) also found. 


\section{Table 3}

Absolute percentage differences in muscle volume (15 mm inter-slice distance) between-sessions (P: preferred limb, NP: non-preferred limb) for two participants. Group muscle volume percentage differences are displayed at the top of each section with percentage differences for the respective individual muscles below*.

\begin{tabular}{|c|c|c|c|c|c|c|c|c|c|c|c|c|}
\hline & $\begin{array}{c}P \\
(\%)\end{array}$ & $\begin{array}{l}\text { NP } \\
(\%)\end{array}$ & $\begin{array}{c}\mathbf{P} \\
(\%)\end{array}$ & $\begin{array}{l}\mathrm{NP} \\
(\%)\end{array}$ & $\begin{array}{c}\mathbf{P} \\
(\%)\end{array}$ & $\begin{array}{l}\text { NP } \\
(\%)\end{array}$ & $\begin{array}{c}P \\
(\%)\end{array}$ & $\begin{array}{l}\text { NP } \\
(\%)\end{array}$ & $\begin{array}{c}P \\
(\%)\end{array}$ & $\begin{array}{l}\text { NP } \\
(\%)\end{array}$ & $\begin{array}{c}\mathbf{P} \\
(\%)\end{array}$ & $\begin{array}{l}\text { NP } \\
(\%)\end{array}$ \\
\hline \multirow[t]{2}{*}{ Hip group } & \multicolumn{2}{|c|}{ Flexors } & \multicolumn{2}{|c|}{ Extensors } & \multicolumn{2}{|c|}{$\begin{array}{l}\text { Internal } \\
\text { rotators }\end{array}$} & \multicolumn{2}{|c|}{$\begin{array}{l}\text { External } \\
\text { rotators }\end{array}$} & \multicolumn{2}{|c|}{ Abductors } & \multicolumn{2}{|c|}{ Adductors } \\
\hline & 3.5 & 0.8 & 1.4 & 0.8 & 2.6 & 0.9 & 2.0 & 0.6 & 4.4 & 2.3 & 2.7 & 0.8 \\
\hline Illiacus & 7.8 & 2.6 & & & & & 7.8 & 2.6 & & & & \\
\hline Gluteus maximus & & & 3.8 & 0.8 & & & 3.8 & 0.8 & 3.5 & 0.8 & 3.5 & 0.8 \\
\hline Gluteus medius & 2.1 & 2.9 & 2.1 & 2.9 & 2.1 & 2.9 & 2.1 & 2.9 & 2.1 & 2.9 & & \\
\hline Gluteus minimus & 10.3 & 9.8 & 1.3 & 9.8 & 10.3 & 9.8 & & & & & & \\
\hline Tensor fasciae latae & 5.8 & 8.3 & & & 5.8 & 8.3 & & & & & 5.8 & 8.3 \\
\hline Piriformis & & & & & & & 14.4 & 15.4 & & & & \\
\hline Gemellus superior & & & & & & & 57.7 & 37.3 & & & & \\
\hline Obturator internus & & & & & & & 13.0 & 12.7 & & & & \\
\hline Gemellus inferior & & & & & & & 27.7 & 39.0 & & & & \\
\hline Obturator externus & & & & & & & 43.9 & 47.1 & & & & \\
\hline Quadratus femoris & & & & & & & 5.1 & 52.2 & & & & \\
\hline Pectineus & 14.1 & 9.6 & & & & & & & & & 14.1 & 9.6 \\
\hline Adductor brevis & 21.0 & 11.3 & & & & & & & & & 21.0 & 11.3 \\
\hline Adductor longus & & & & & & & & & & & 9.1 & 8.2 \\
\hline Adductor magnus & & & 1.2 & 2.5 & & & & & & & 1.2 & 2.5 \\
\hline Gracilis & 1.3 & 4.6 & & & 1.3 & 4.6 & & & & & 1.3 & 4.6 \\
\hline Sartorius & 4.5 & 3.3 & & & & & 4.5 & 3.3 & 4.5 & 3.3 & & \\
\hline \multirow[t]{2}{*}{ Knee group } & \multicolumn{2}{|c|}{ Flexors } & \multicolumn{2}{|c|}{ Extensors } & \multicolumn{2}{|c|}{$\begin{array}{l}\text { Internal } \\
\text { rotators }\end{array}$} & \multicolumn{2}{|c|}{$\begin{array}{l}\text { External } \\
\text { rotators }\end{array}$} & & & & \\
\hline & 1.0 & 0.7 & 1.0 & 1.4 & 0.4 & 2.8 & 5.9 & 3.5 & & & & \\
\hline Semitendinosus & 4.1 & 3.4 & & & 4.1 & 3.4 & & & & & & \\
\hline $\begin{array}{l}\text { Biceps femoris long } \\
\text { head }\end{array}$ & 7.9 & 4.3 & & & & & 7.9 & 4.3 & & & & \\
\hline $\begin{array}{l}\text { Biceps femoris } \\
\text { short head }\end{array}$ & 1.6 & 2.4 & & & & & 1.6 & 2.4 & & & & \\
\hline Semimembranosus & 1.9 & 3.5 & & & 1.9 & 3.5 & & & & & & \\
\hline Rectus femoris & & & 0.9 & 3.5 & & & & & & & & \\
\hline Vastus lateralis & & & 5.2 & 2.2 & & & & & & & & \\
\hline Vastus intermedialis & & & 5.2 & 0.2 & & & & & & & & \\
\hline Vastus medialis & & & 2.6 & 1.0 & & & & & & & & \\
\hline Popliteus & 14.3 & 10.6 & & & & & & & & & & \\
\hline \multirow[t]{2}{*}{ Ankle group } & \multicolumn{2}{|c|}{ Dorsiflexors } & \multicolumn{2}{|c|}{ Plantarflexors } & \multicolumn{2}{|c|}{ Evertors } & \multicolumn{2}{|c|}{ Invertors } & & & & \\
\hline & 0.5 & 2.1 & 1.0 & 4.9 & 1.6 & 0.3 & 0.3 & 8.9 & & & & \\
\hline
\end{tabular}




\begin{tabular}{|c|c|c|c|c|c|c|c|c|}
\hline $\begin{array}{l}\text { Medial } \\
\text { gastrocnemius }\end{array}$ & & & 1.4 & 1.9 & & & & \\
\hline $\begin{array}{l}\text { Lateral } \\
\text { gastrocnemius }\end{array}$ & & & 6.1 & 15.7 & & & & \\
\hline Soleus & & & 1.4 & 10.1 & & & & \\
\hline Tibialis anterior & 1.7 & 4.2 & & & & & 1.7 & 4.2 \\
\hline $\begin{array}{l}\text { Extensor digitorum } \\
\text { and hallucis longus }\end{array}$ & 1.6 & 0.6 & & & 1.6 & 0.6 & & \\
\hline Peroneals & & & 1.7 & 0.9 & 1.7 & 0.9 & & \\
\hline Tibialis posterior & & & 2.0 & 4.0 & & & 2.0 & 4.0 \\
\hline $\begin{array}{l}\text { Flexor digitorum } \\
\text { longus }\end{array}$ & & & 19.8 & 6.0 & & & 19.8 & 6.0 \\
\hline $\begin{array}{l}\text { Flexor hallucis } \\
\text { longus }\end{array}$ & & & 4.9 & 26.9 & & & 4.9 & 26.9 \\
\hline
\end{tabular}

*The hip flexor group includes rectus femoris. The hip extensor group includes semitendinosus, biceps

femoris long head, and semimembranosus. The knee flexor group includes the medial and lateral

gastrocnemii. 
Establishing normative muscle size and symmetry

Similar total MV was found in both limbs (mean MV P: $66.4 \pm 4.9 \mathrm{~cm}^{3} / \mathrm{kg} . \mathrm{m}$, NP: 66.3 $\left.\pm 4.5 \mathrm{~cm}^{3} / \mathrm{kg} . \mathrm{m}\right)$. Mean SI was $0.4 \pm 4.1 \%$ and mean ASI was $12.9 \pm 2.6 \%$. The only muscle to show both practically and statistically significant between-limb differences across all participants was popliteus (ASI: $18.1 \pm 9.4 \%$, effect size: $0.93, p=0.047$ ) although practically significant differences were found for gemellus superior (effect size: 0.73), gemellus inferior (effect size: 0.66), and biceps femoris short head (effect size: 0.73). Individual MV, SI and ASI are presented in Figure 1. 


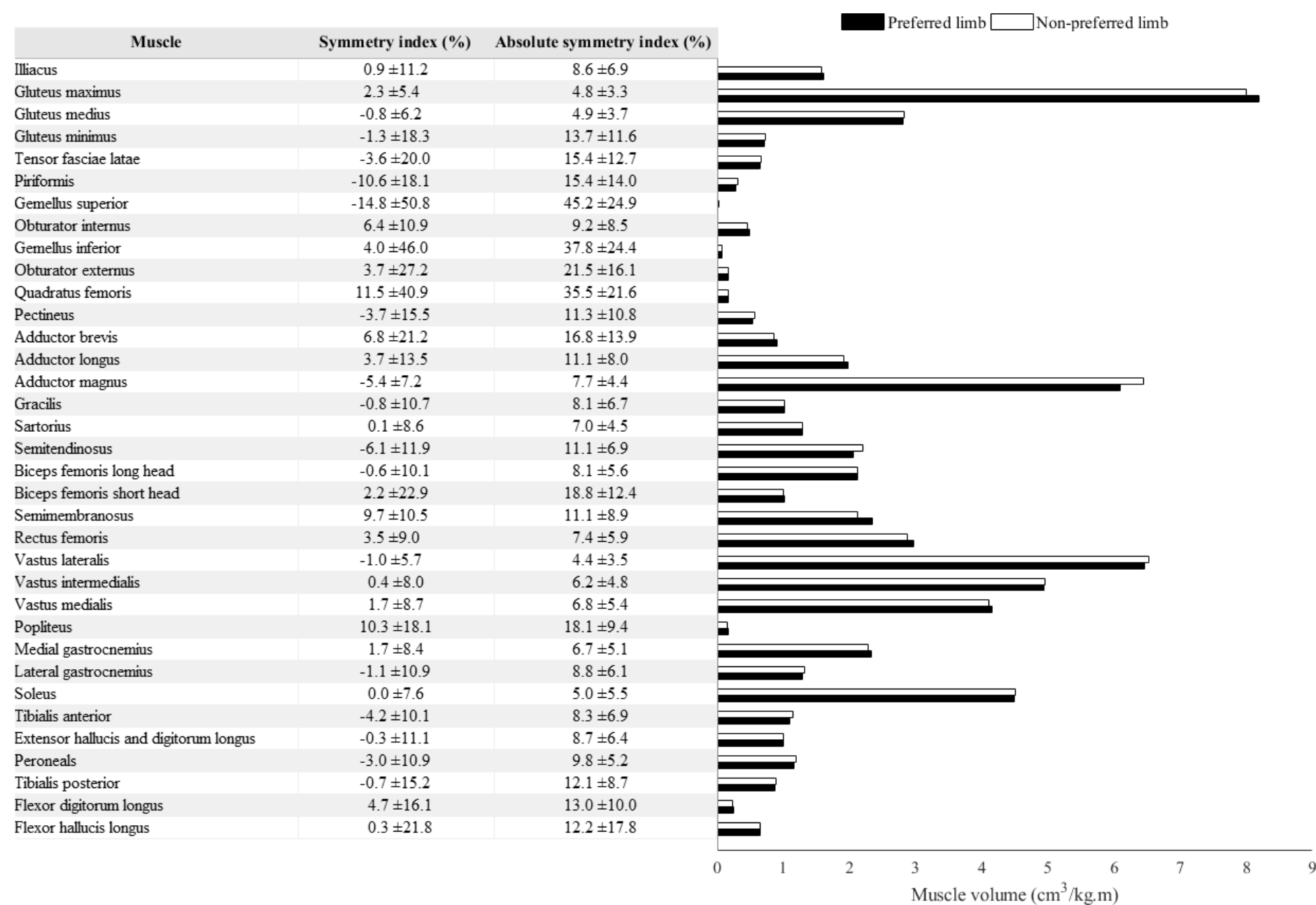

Figure 1: Mean individual muscle volume (15 mm inter-slice distance), symmetry index and absolute symmetry index. Practically and statistically significant between-limb differences in muscle volume were observed for popliteus only. 
Between-limb MV differences were reduced when evaluated as functional anatomical groups (mean SI: $0.0 \pm 1.2 \%$, mean ASI: $4.7 \pm 1.8 \%$ ). Differences were not statistically or practically significant for any group. Group MV, SI and ASI are presented in Figure 2. 


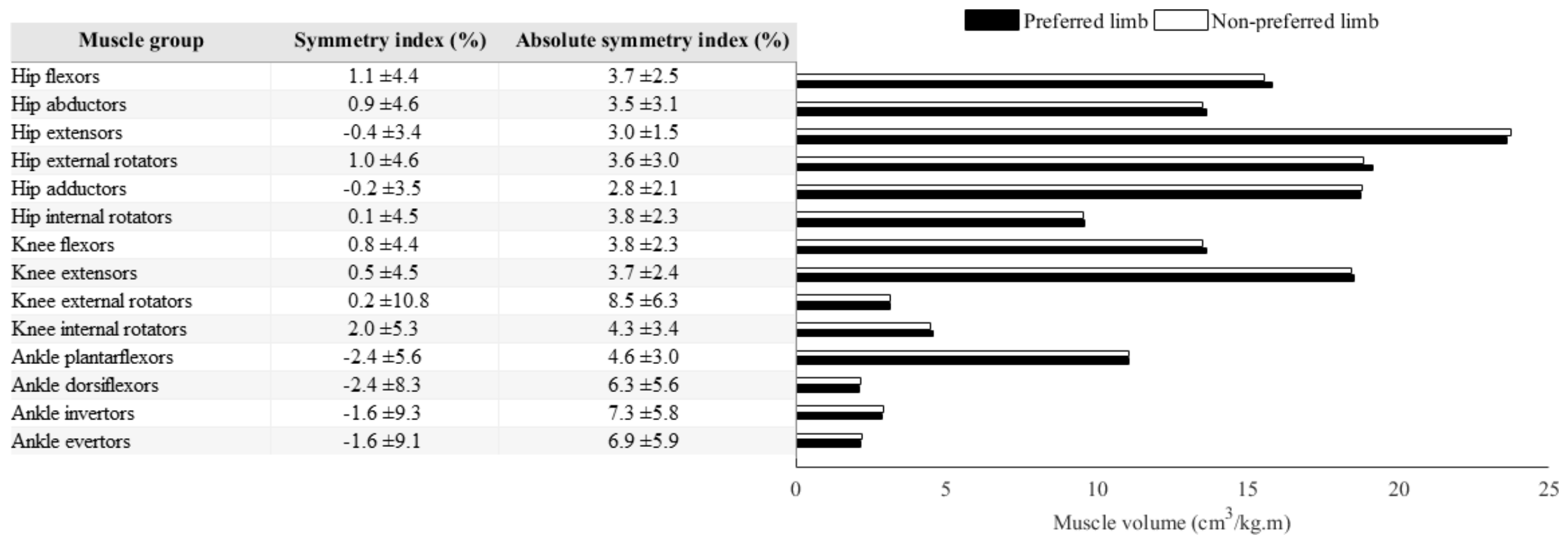

Figure 2: Mean functional anatomical group volume (15 mm inter-slice distance), symmetry index and absolute symmetry index. No practically or statistically significant between-limb differences in muscle volume were observed 
The pattern of individual MV z-scores throughout the lower limb differed across individuals (Figure 3). When an individual had large MV, this tended to be the case in both limbs. Notable exceptions were rectus femoris in participants nine (z-score P: 0.74, NP: 1.43) and twelve (z-score P: 2.04, NP: 0.16), gracilis in participant twelve (z-score P: 1.64, NP: 0.81), and vastus medialis in participant thirteen (z-score P: 1.49, NP: $0.83)$. 


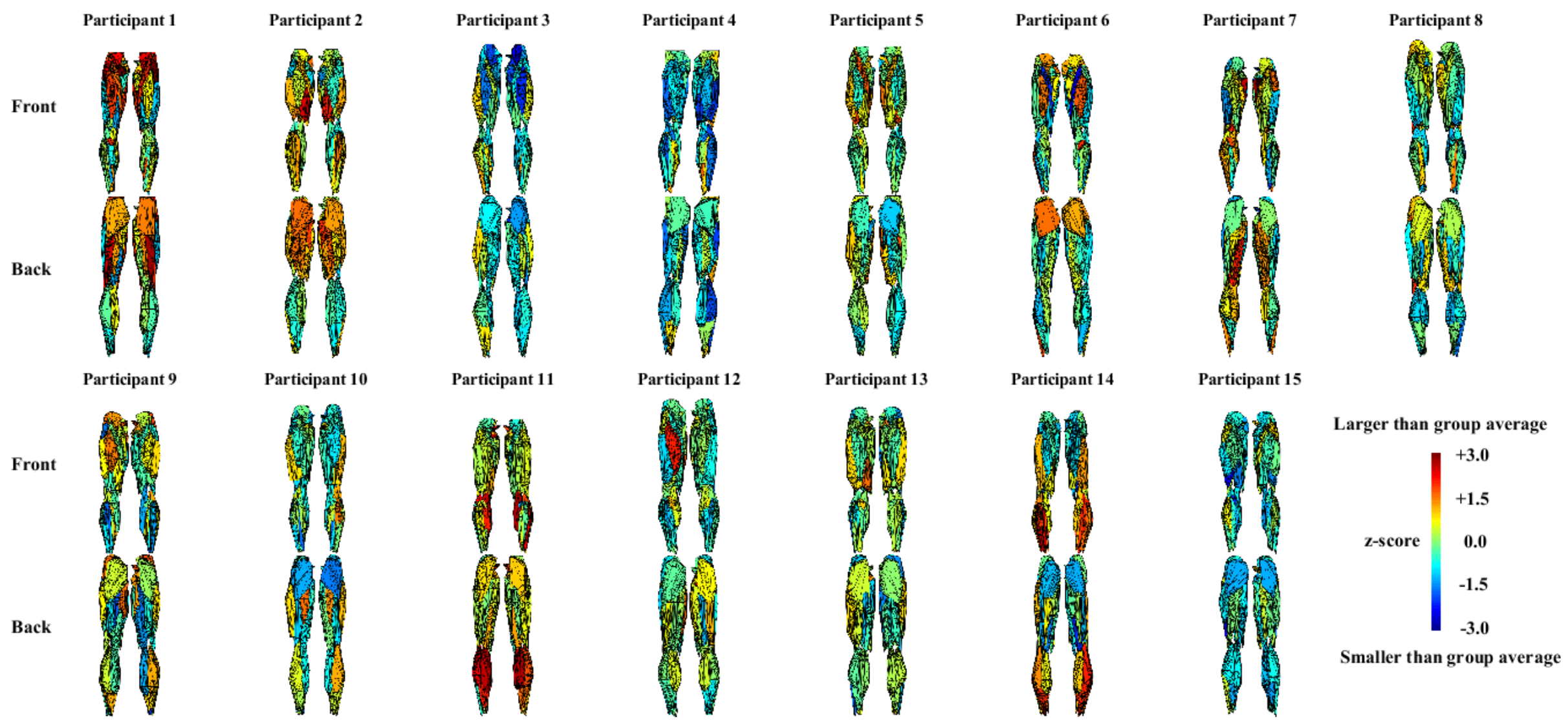

Figure 3: Individual participant muscle size relative to the group mean. The front and back of the right and left limb are displayed below one another. Participants 1, 2, 3, and 9 preferred their left limb for kicking and all other participants preferred their right 


\section{DISCUSSION}

This study aimed to evaluate the application of MRI for measuring bilateral lower limb muscle size and symmetry in healthy adult males, with a specific focus on decreasing analysis time, establishing measurement reliability, and quantifying typical muscle size and size asymmetry in healthy adult males. Use of an inter-slice distance of $15 \mathrm{~mm}$ considerably reduced the analysis time required and was appropriate for application across most muscles. However, the data supports the application of a larger inter-slice distance for longer muscles and taller individuals. Smaller inter-slice distances should be used for shorter muscles and people, e.g. in paediatric populations. Manual measurement of muscle size from MRI images was reliable, particularly when reported using MV. CSA measurement reliability was good for large CSA and reduced when CSA were smaller or the time between repeated measurements was increased. Healthy adult males showed small between-limb differences in MV although this was not achieved in the same way across individuals.

The difference in MV calculated with a $15 \mathrm{~mm}$ inter-slice distance, compared to the reference method, was on average less than $1.0 \%$ and offered a considerable time saving. Increasing inter-slice distance to $20 \mathrm{~mm}$ increased the percentage difference for muscles with important functional roles such as gluteus medius (absolute difference 15 mm: $0.8 \pm 0.5 \%, 20 \mathrm{~mm}: 1.6 \pm 1.4 \%$ ) and lateral gastrocnemius (absolute difference 15 $\mathrm{mm}: 1.0 \pm 0.8 \%, 20 \mathrm{~mm}: 2.0 \pm 1.9 \%)$. The time saved when using a $15 \mathrm{~mm}$ inter-slice distance was significant as the time demand of analysing all 70 muscles across both legs of one participant every $15 \mathrm{~mm}$ (i.e. 24 hours) was equivalent to analysing the gluteals, quadriceps, hamstrings, and triceps surae of both legs (i.e. 28 muscles) every $5 \mathrm{~mm}$. The absolute percentage difference in MV increased with further increases in inter-slice distance for all muscles with the exception of the quadriceps, where increasing inter-slice distance up to $30 \mathrm{~mm}$ showed minimal percentage differences but offered a large reduction in time demand (Table 1). This is likely to be due to the quadriceps being a long muscle group with a regular and consistent shape and supports previous research suggesting an inter-slice distance of 30 to $36 \mathrm{~mm}$ can estimate quadriceps MV with sufficient accuracy (Nordez et al. 2009). Use of larger inter-slice distances than $15 \mathrm{~mm}$ may be appropriate for longer muscles and taller individuals although this was not appropriate for application throughout the lower limb in the 
current study. A $15 \mathrm{~mm}$ inter-slice distance may be considered too large for some muscles such as gluteus minimus, due to its short length (mean length P: $11.9 \pm 2.0 \mathrm{~cm}$, NP: $11.2 \pm 1.4 \mathrm{~cm}$ ) and irregular shape, both of which should be considered when selecting an appropriate inter-slice distance. The current study used a $15 \mathrm{~mm}$ inter-slice distance uniformly throughout the lower limb, with the exception of the small hip external rotators, due to the resulting considerable reduction in time demand and negligible increase in percentage difference compared to the reference method. However, although key muscle groups such as the quadriceps can be manually analysed in a relatively short period of time, a comprehensive and accurate analysis of the entire lower limb remains a time-intensive process. Further reductions may be achieved by combining manual analysis with semi-automated muscle boundary identification image processing techniques, or potentially exploring the upper limit of increasing inter-slice distance when using cubic spline interpolation to estimate intermediate slices as has been conducted previously in the quadriceps (Nordez et al. 2009). However, similar to the approach presented here, the effects of increasing inter-slice distances with spline approaches are also likely to be affected by the height of an individual, or an individual muscle's shape.

Manual analysis of muscle CSA showed good measurement reliability within- and between-sessions in general. The ICC reported compare to previously established intrainvestigator reliability in the individual quadriceps, hamstrings, sartorius, gracilis, tensor fascia latae, medial and lateral gastrocnemii, and soleus (ICC: $0.95-0.99$, Tate et al. 2006). The lowest ICC were found between-sessions over six months for vastus medialis bilaterally at $25 \%$ of thigh length (P: 0.63, NP: 0.62), and TE values were larger than for other muscles in the analysis (P: $0.9 \mathrm{~cm}^{2}, 14.8 \%, \mathrm{NP}: 1.2 \mathrm{~cm}^{2}, 22.4 \%$ ). This may be attributable to CSA size as larger CSA at $75 \%$ of thigh length showed good reliability (ICC P: 0.98, NP: 0.97; TE P: $0.2 \mathrm{~cm}^{2}, 0.6 \%$, NP: $0.4 \mathrm{~cm}^{2}, 1.3 \%$ ). Throughout the reliability analysis, small CSA showed poorer reliability. The largest absolute TE of these was $1.2 \mathrm{~cm}^{2}$ for NP vastus medialis at $25 \%$ of thigh length, where the mean CSA was $5.4 \pm 2.9 \mathrm{~cm}^{2}$. This highlights a potential limitation in using CSA alone to identify changes in muscle size.

Muscle volume showed good between-session measurement reliability in the current study, with the exception of smaller muscles in areas where boundaries can be difficult 
to identify, such as gluteus minimus, adductor brevis, popliteus, flexor hallucis longus, and flexor digitorum longus. Large between-session differences were also found for the small hip external rotators, possibly due to their orientation in the oblique plane, and this should be considered in future research utilising MRI images of these muscles. The percentage differences recorded for larger muscles and groups do however support the use of MRI derived MV for quantifying muscle hypertrophy (Balshaw et al. 2017) or atrophy (Feger et al. 2016). Small between-session percentage differences were found for functional anatomical groups, possibly because discrepancies in muscle boundary identification between muscles in close proximity were accounted for when individual MV were summed together. As muscles function as groups this may be a preferable representation of MV in future research. However, this should be approached by summing individual MV, as in the current study, to account for inter-muscular adipose and connective tissue which can lead to systematic overestimation of volume if included in MV calculations (mean difference in MV when using group compared to individual CSA P: $8.3 \pm 3.6 \%$, NP: $10.9 \pm 3.5 \%$ ).

To the authors' knowledge, this is the first published comprehensive dataset of bilateral MV in recreationally active adult males. Total MV (P: $66.4 \pm 4.9 \mathrm{~cm}^{3} / \mathrm{kg} . \mathrm{m}, \mathrm{NP}: 66.3$ $\left.\pm 4.5 \mathrm{~cm}^{3} / \mathrm{kg} . \mathrm{m}\right)$ was larger than previously reported in healthy males and females $(58.0$ $\pm 4.2 \mathrm{~cm}^{3} / \mathrm{kg} . \mathrm{m}$, Handsfield et al. 2014) and smaller than in collegiate sprinters $(71.0$ $\pm 6.4 \mathrm{~cm}^{3} / \mathrm{kg} . \mathrm{m}$, Handsfield et al. 2017). This demonstrates a continuum of training related hypertrophy, as although all were active at least three days per week (as per definition of recreationally active), several participants were involved in activity most days of the week. However, the inclusion of females in both prior studies, and omission of psoas due to the imaging protocol used in the current study, prevents a true comparison. A combined volume of the quadriceps, hamstrings and triceps surae in the current study (mean volume P: $34.2 \pm 2.4 \mathrm{~cm}^{3} / \mathrm{kg} . \mathrm{m}$, NP: $34.0 \pm 2.5 \mathrm{~cm}^{3} / \mathrm{kg} . \mathrm{m}$ ) was similar to previously reported in an all-male population of elite sprinters $(32.8$ $\left.\mathrm{cm}^{3} / \mathrm{kg} . \mathrm{m}\right)$ and endurance runners $\left(31.3 \mathrm{~cm}^{3} / \mathrm{kg} . \mathrm{m}\right.$, Bex et al. 2017), although the previous study did not provide hip extensor volumes which are important for comparison due to their functional role as the main producers of power in the lower limb. 
Minimal between-limb differences in individual MV have been identified (mean SI: 0.4 $\pm 4.1 \%$, ASI: $12.9 \pm 2.6 \%$ ). Positive and negative SI for individual muscles and functional anatomical groups suggest symmetry was not consistently influenced by limb preference, in contrast to findings in athletes from sports with a change of direction and/or jumping requirement (Tate et al. 2006). The ASI observed for most muscles and groups in the current study was larger than between-session MV percentage differences, although the only muscle showing both practically and statistically significant betweenlimb differences was popliteus, and relatively large between-session differences in MV (P: $14.3 \%$, NP: $10.6 \%$ ) may have contributed to this. Given that participants were free from injury, had no history of lower limb surgery, and were recreationally active at the time of the study, these results provide a typical measurement of baseline muscle size and symmetry for comparison and utilisation in future research investigating the role of muscle function in performance.

Across participants, a larger range of ASI was observed for individual muscles $(0.1 \%$ to $167.5 \%)$ compared to functional anatomical groups $(0.1 \%$ to $44.9 \%)$. This is an important consideration for training and rehabilitation programme design as muscles are trained in groups, rather than in isolation. However, the contribution of some muscles to multiple, sometimes antagonistic, movements provides a methodological consideration. For example, gluteus maximus is a hip abductor and adductor through its upper and lower fibres, respectively. Similarly, extensor digitorum longus everts and extensor hallucis longus inverts the ankle (Floyd 2007). Due to difficulties distinguishing boundaries, both have been represented as one muscle in the current study, as in previous studies (Handsfield et al. 2014, 2017). This area of research would benefit from additional work establishing appropriate partitioning of lower limb muscles so measurements of muscle group size and symmetry can be more closely associated with functional anatomical movements and performance.

The variety of muscle size relative to the group mean observed across participants may reflect a multifaceted hypertrophic response to the diversity of recreational activity (Figure 3). Participants 11 and 14 reported being recreational runners and both displayed larger shank muscles. Soleus in particular was larger than reported in elite endurance runners (Bex et al. 2017), likely reflective of a reduced endurance training volume in the current study, resulting in a larger MV. Rectus femoris was larger than 
the group mean unilaterally in participants nine and twelve, as was gracilis in participant twelve and vastus medialis in participant thirteen. The reduction in betweenlimb differences observed when muscles were represented as part of their functional anatomical groups suggests this is a typical trait of healthy symmetry.

This study has identified $15 \mathrm{~mm}$ as an appropriate inter-slice distance for considerably reducing the time-demand of estimating MV of the lower limb muscles using manual analysis of MRI images. To ensure measurement accuracy it is recommended that smaller muscles such as the small hip external rotators, gluteus minimus, popliteus, flexor hallucis longus, and flexor digitorum longus are analysed on every available slice (i.e. every $5 \mathrm{~mm}$ ). Manual analysis of MRI images has shown good measurement reliability and MV provided a more reliable between-session measurement of muscle size than CSA, an important consideration when identifying changes in muscle size between individuals and timepoints. Typical muscle size and symmetry have been quantified in healthy adult males, with small between-limb differences in muscle size observed. Between-limb differences in MV were minimal when muscles were observed in functional anatomical groups based on their agonistic actions. This is an important consideration when using muscle size as a measurement of symmetry and initial between-limb comparisons should be conducted using muscle group volumes.

\section{Acknowledgements:}

This research was funded by Loughborough University as part of Dr. Laura-Anne M Furlong's start-up package, and the Engineering and Physical Sciences Research Council via the Loughborough University Mini Centre for Doctoral Training in Defence Medicine (EP EP/N509516/1). 


\section{REFERENCES}

Balshaw T G, Massey G J, Maden-Wilkinson T M, Morales-Artacho A J, McKeown A, Appleby C L, Folland J P 2017 Changes in agonist neural drive, hypertrophy and pre-training strength all contribute to the individual strength gains after resistance training European Journal of Applied Physiology 117 631-640

Bennett D L 2016 Stanford musculoskeletal MRI atlas, Stanford MSK MRI Atlas http://www.xrayhead.com

Bex T, Iannaccone F, Stautemas J, Baguet A, De Beule M, Verhegge B, Aerts P, De Clercq D, Derave W 2017 Discriminant musculo-skeletal leg characteristics between sprint and endurance elite Caucasian runners Scandinavian Journal of Medicine and Science in Sports 27 275-281

Cohen J 1988 Statistical Power Analysis for the Behavioral Sciences. $2^{\text {nd }}$ Edition. (Hillsdale, NJ: Lawrence Erlbaum Associates, Inc., Publishers)

Evangelidis P E, Massey G J, Pain M T G, Folland J P 2016 Strength and size relationships of the quadriceps and hamstrings with special reference to reciprocal muscle balance European Journal of Applied Physiology 116 593-600

Feger M A, Snell S, Handsfield G G, Blemker S S, Wombacher E, Fry R, Hart J M, Saliba S A, Park J S, Hertel J 2016 Diminished foot and ankle muscle volumes in young adults with chronic ankle instability Orthopaedic Journal of Sports Medicine 4 $1-8$

Floyd R T 2007 Manual of Structural Kinesiology. 16th edn. (New York, NY: McGraw-Hill)

Fuggle N, Shaw S, Dennison E, Cooper C 2017 Sarcopenia Best Practice \& Research Clinical Rheumatology 31 218-242

Grimaldi A, Richardson C, Durbridge G, Donnelly W, Hides J 2009 The association 
between degenerative hip joint pathology and size of the gluteus medius, gluteus minimus and piriformis muscles Manual Therapy 14 605-610

Handsfield G G, Meyer C H, Hart J M, Abel M F, Blemker S S 2014 Relationships of 35 lower limb muscles to height and body mass quantified using MRI Journal of Biomechanics 47 631-638

Handsfield G G, Meyer C H, Abel M F, Blemker S S 2016 Heterogeneity of muscle sizes in the lower limbs of children with cerebral palsy Muscle and Nerve 53 922-945

Handsfield G G, Knaus K R, Fiorentino N M, Meyer C H, Hart J M, Blemker S S 2017 Adding muscle where you need it: non-uniform hypertrophy patterns in elite sprinters Scandinavian Journal of Medicine and Science in Sports 27 1050-1060

Hopkins W G, Marshall S W, Batterham A M, Hanin J 2009 Progressive statistics for studies in sports medicine and exercise science Medicine and Science in Sports and Exercise 41 3-12

Karamanidis K, Arampatzis A, Bruggemann G-P 2003 Symmetry and reproducibility of kinematic parameters during various running techniques Medicine and Science in Sports and Exercise 35 1009-1016

Nordez A, Jolivet E, Studhoff I, Bonneau D, De Guise J A, Skalli W 2009 Comparison of methods to assess quadriceps muscle volume using magnetic resonance imaging Journal of Magnetic Resonance Imaging 30 1116-1123

Tate C M, Williams G N, Barrance P J, Buchanan T S 2006 Lower extremity muscle morphology in young athletes: An MRI-based analysis Medicine and Science in Sports and Exercise 38 122-128 